\title{
Cross-linked xenogenic collagen implantation in the sheep model for vaginal surgery
}

\author{
Masayuki Endo • Iva Urbankova • Jaromir Vlacil • \\ Siddarth Sengupta $\cdot$ Thomas Deprest • \\ Bernd Klosterhalfen • Andrew Feola • Jan Deprest
}

Received: 25 September 2014 / Accepted: 19 January 2015 / Published online: 5 February 2015

(C) The Author(s) 2015. This article is published with open access at Springerlink.com

\begin{abstract}
The properties of meshes used in reconstructive surgery affect the host response and biomechanical characteristics of the grafted tissue. Whereas durable synthetics induce a chronic inflammation, biological grafts are usually considered as more biocompatible. The location of implantation is another determinant of the host response: the vagina is a different environment with specific function and anatomy. Herein, we evaluated a cross-linked acellular collagen matrix (ACM), pretreated by the anti-calcification procedure ADAPT ${ }^{\circledR}$ in a sheep model for vaginal surgery. Ten sheep were implanted with a cross-linked $\mathrm{ACM}$, and six controls were implanted with a polypropylene $\left(\mathrm{PP} ; 56 \mathrm{~g} / \mathrm{m}^{2}\right.$ ) control. One implant was inserted in the lower rectovaginal septum, and one was used for abdominal wall defect reconstruction. Grafts were removed after 180 days; all graftrelated complications were recorded, and explants underwent bi-axial tensiometry and contractility testing. Half of ACMimplanted animals had palpable induration in the vaginal implantation area, two of these also on the abdominal implant. One
\end{abstract}

M. Endo $\cdot$ I. Urbankova $\cdot$ J. Vlacil $\cdot$ A. Feola $\cdot$ J. Deprest

Centre for Surgical Technologies, Faculty of Medicine, KU Leuven,

Herestraat 49, 3000 Leuven, Belgium

M. Endo $\cdot$ I. Urbankova $\cdot$ J. Vlacil $\cdot$ S. Sengupta $\cdot$ T. Deprest $\cdot$

A. Feola $\cdot$ J. Deprest $(\bowtie)$

Department of Development and Regeneration, Organ Systems

Cluster, Faculty of Medicine, KU Leuven, Herestraat 49,

3000 Leuven, Belgium

e-mail: Jan.Deprest@uzleuven.be

M. Endo $\cdot$ J. Deprest

Pelvic Floor Unit, University Hospitals KU Leuven,

Leuven, Belgium

I. Urbankova $\cdot$ J. Vlacil

Institute for Care of Mother and Child, Prague, Czech Republic

B. Klosterhalfen

Institute for Pathology, Düren Hospital, Düren, Germany animal had a vaginal exposure. Vaginal ACMs were $63 \%$ less stiff compared to abdominal ACM explants $(p=0.01)$ but comparable to vaginal PP explants. Seven anterior vaginal ACM explants showed areas of graft degradation on histology. There was no overall difference in vaginal contractility. Considering histologic degradation in the anterior vaginal implant as representative for the host, posterior ACM explants of animals with degradation had a $60 \%$ reduced contractility as compared to PP $(p=0.048)$. Three abdominal implants showed histologic degradation; those were more compliant than nondegraded implants. Vaginal implantation with ACM was associated with graft-related complications (GRCs) and biomechanical properties comparable to PP. Partially degraded ACM had a decreased vaginal contractility.

Keywords Graft-related complication · Biological graft . Prolapse $\cdot$ Biomechanics $\cdot$ Contractility

\section{Introduction}

Pelvic organ prolapse (POP) develops in half of parous women over 50 years, half of them being symptomatic with only $20 \%$ of them seeking medical help $[1,2]$. Lifetime risk of POP surgery is $19 \%$ [3], and up to $25 \%$ require later re-operation [4]. It was suggested that this may be reduced by using synthetic or biological implants $[4,5]$. Although durable synthetic meshes are known to achieve good anatomical and functional results for cystocele repair, they may cause graft-related complications (GRCs) in over $10 \%$ of women [4]. Alternative grafts that may reduce the number of GRC yet still provide durable results could be contemplated [6-10].

Biological grafts are derived from either human (autograft or allografts) or animal material (xenografts). Autografts, such 
as fascia lata, inherently have donor site-related morbidity and also have unpredictable durability $[11,12]$. Allografts are retrieved from cadaveric tissue, and although fastidious steps are taken in preparation, concerns regarding transmission of possible prion disease or viruses remained. Xenografts are acellular collagen matrices (ACMs) that are either derived from the dermis, pericardium or small intestinal submucosa of animals that are purposely bred in strictly controlled conditions. Most ACMs are of bovine or porcine origin, which, during their production, undergo various chemical procedures (cross-linking, sterilization). After implantation, ACMs are remodelled and/or replaced by connective tissue within variable time periods. The latter can be modified using, e.g. cross-linking agents which leads to the formation of excessive intramolecular and intermolecular chemical bonds preventing decomposition by endogenous collagenases [13]. This alters the properties of the ACM either physically or chemically. A commonly used cross-linking agent is glutaraldehyde (GAD) [13] resulting in durable grafts that are slowly integrated and remodelled. However, residual GAD is cytotoxic and may cause calcification. To prevent this, Neethling et al. developed a multi-step anti-mineralization process called ADAPT ${ }^{\circledR}[14]$. This enhances crosslink stability, removes residual GAD and modifies the nonbifunctionally reacted GAD residues. The process reduces lipid content and restores tissue elasticity [14]. ADAPT ${ }^{\circledR}$-treated bovine pericardial patches have been successfully used in surgery of congenital heart defects without demonstrable calcification in a 36-month follow-up period [15]. These promising results demonstrating long-term stability in challenging circumstances are worthwhile considering for translation in pelvic floor surgery. Herein, we used ADAPT $^{\circledR}$-treated xenografts in a sheep model for transvaginal surgery, studying both the occurrence of GRCs and active and passive biomechanical properties of the vaginal wall. As a reference, we compared outcomes to repairs with light weight polypropylene (PP) implants.

\section{Materials and methods}

\section{Implants and surgery}

This study compares outcomes following vaginal and abdominal mesh insertion of either a xenogenic or synthetic implant in a sheep model. The xenogenic graft was a non-perforated acellular collagen matrix (ACM) derived from bovine pericardium which was cross-linked with an ultra-low concentration $(0.05 \%)$ of monomeric GAD. Further preimplantation processing included the so-called ADAPT ${ }^{\circledR}$ anti-calcification procedure and sterilization with propylene oxide [14, 16] (material donated by Prof WML Neethling, Fremantle, Australia). As a control, we used a commercially available monofilament polypropylene (PP) mesh used for vaginal prolapse repair (PP) (Avaulta Solo; $56 \mathrm{~g} / \mathrm{m}^{2}$, Bard Medical, Covington, GA, USA). The latter was purchased and delivered sterile via the hospital pharmacy. Observations of these animals were earlier reported on elsewhere [17].

The anaesthetic, surgical technique and methodology used for outcome evaluation have been described in detail previously $[18,19]$. Briefly, 16 parous Texel sheep (mean weight $68 \pm$ $3.5 \mathrm{~kg}$ ) were obtained from the Zootechnical Centre of the KU Leuven. They underwent simultaneous vaginal and abdominal implantation with either ACM $(N=10)$ or PP $(N=6)$. Surgery was conducted in sterile conditions under general anaesthesia with prophylactic antibiotics at induction and 3 days of postoperative analgesia. Following aqua dissection, a single incision was made in the recto-vaginal septum that was then dissected to create a suitable space for a $35 \times 35 \mathrm{~mm}$ suture fixed prosthesis (posterior implant) (Fig. 1). Additionally, a $10 \times 20 \mathrm{~mm}$ graft was inserted in the anterior vaginal wall (anterior implant). Finally, a $50 \mathrm{~mm}$ longitudinal paramedian cutaneous incision was made in the anterior abdominal wall, and a $40-\mathrm{mm}$ primarily suture repaired full-thickness fascial incision was overlaid with the same graft as used vaginally (Fig. 1). All implants were fixed with interrupted PP $4 / 0$ prolene sutures (Ethicon). Postoperatively, animals were allowed to move, drink and eat ad libitum and were clinically followed by a veterinarian.

\section{Outcome measures}

On average, 180 days later, animals were euthanized, and during necropsy graft-related complications (GRCs) or the presence of herniation was noted at each of the three implantation sites. Thereafter, the original implant together with the adjacent and ingrown tissue (further referred to as explant) was removed "en bloc" and its dimensions and thickness were determined as an average of three random measurements with a digital micrometer (Mitutoyo, Kawasaki, Japan; accuracy $0.01 \mathrm{~mm}$ ). Contraction of the explant was defined as the explant area over initial graft area $\left(1225 \mathrm{~mm}^{2}\right.$ for vaginal, $2500 \mathrm{~mm}^{2}$ for abdominal implant, respectively). Explants were then divided to obtain specimens for histology and biomechanical testing as shown in Fig. 1. For vaginal explants, the anterior specimens were used for histology while the posterior explants were used for active and passive biomechanical testing. The larger abdominal explants were divided for both histology and passive biomechanics.

Histology quantified the inflammatory response and connective tissue formation on 5 - $\mu$ m-thick sections, stained with hematoxylin and eosin $(\mathrm{H} \& \mathrm{E})$ and Movat pentachrome, using an ordinal scoring system [20, 21]. Two operators blinded to the initial treatment counted foreign body giant cell (FBGC), polymorphonuclear (PMN) cells, newly formed vessels and collagen organization, composition and amount in five randomly chosen areas at the implant-host 
Fig. 1 Schematic drawing of abdominal (a) and vaginal (b) implantation in the sheep model. Specimens explanted (c) from the abdomen and anterior (ant) and posterior (post) vaginal wall were divided according with their respective testing method. The arrow is pointing cranially in the direction to the uterine cervix (illustration by Myrthe Boymans)

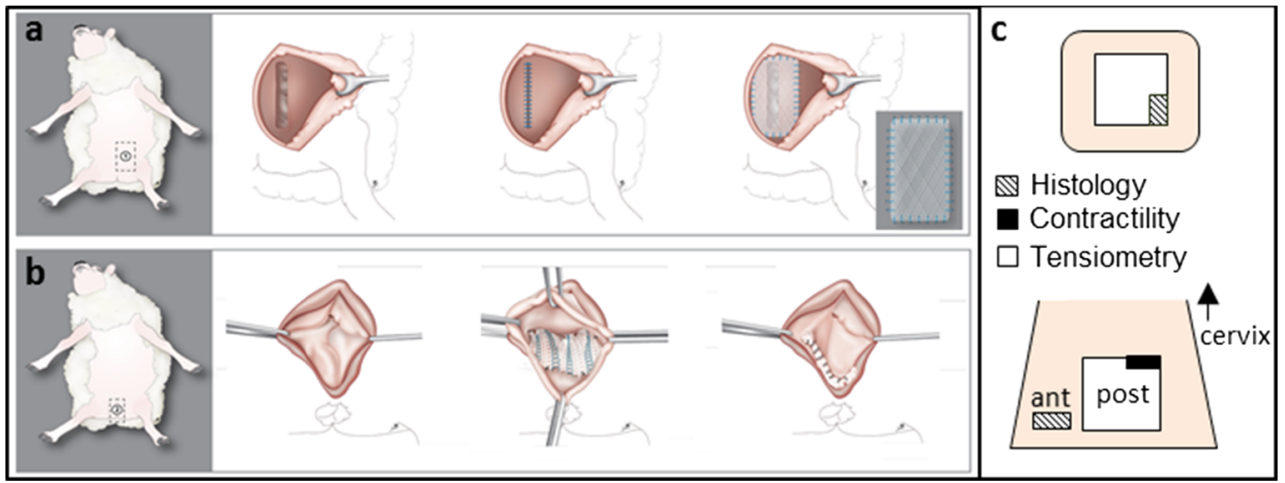

tissue interface at a magnification $\times 400$ (Zeiss Axioplan 400, Oberkochen, Germany). Infection was classified as either low grade ( $\geq 15$ PMN per HPF and no clinical evidence of infection) or high grade (presence of micro-abscesses, dense inflammatory infiltrate, fibrin exudation, bleeding and necrosis as well as clinical signs of infection, if any) [22]. All histological scores (FBGCs, PMN, vessel and collagen scores) were at least done in duplicate and averaged.

Passive biomechanics were tested using bi-axial tensiometry by a plunger test on a 500-N Zwick tensiometer with a $200-\mathrm{N}$ cell load (Zwick GmbH \& Co. KG, Ulm, Germany) using a protocol defined earlier [18]. A spherical $11.5 \mathrm{~mm}$ plunger was passed through an aperture $\varnothing 20 \mathrm{~mm}$ that exposing the explant compressed in $\varnothing 30-\mathrm{mm}$ rings. All explants were placed with the graft facing upward. Records of the force-elongation relationship allowed to define the stiffness $(\mathrm{N} / \mathrm{mm})$ of the tested material, which we measured in the low-stress area also called as the "comfort" zone, the average slope of the forceelongation curve and the length of the former zone. These values were determined by TestXpert II software (Zwick $\mathrm{GmbH} \& \mathrm{Co}$ ). Active biomechanics of a circumferentialoriented posterior vaginal explant as well as control strip $(10 \times 8 \mathrm{~mm})$ were assessed by a contractility assay (Fig. 1) [23]. This is an ex vivo assessment quantifying the ability of the smooth muscles present in vaginal tissue strips to contract by immersing them in an organ bath at $80 \mathrm{mM} \mathrm{KCl}$ concentration $\left(\mathrm{mN} / \mathrm{mm}^{3}\right)$, suggested as a proxy for vaginal function.

Statistical analysis and ethics committee approval

Data are reported as mean and standard deviation or as median and interquartile range depending on the distribution. Either a Student's $t$ test or a Mann-Whitney $U$ test was performed to compare PP and ACM. Chi-square was used for categorical data. Pairs of abdominal and vaginal explants from the same animal were also tested using a paired $t$ test or Wilcoxon signed-rank test. All analyses were performed with Prism 5 (GraphPad Software, Inc., La Jolla, CA, USA), and the significance level was set up to $p<0.05$. Animals were housed in controlled conditions and treated in accordance with current national guidelines on animal welfare. The experiment was approved by the Ethics Committee for Animal Experimentation of the Faculty of Medicine of the KU Leuven.

\section{Results}

Vaginal versus abdominal implantation of ACM

Five out of ten sheep implanted with ACM developed GRCs at the vaginal implantation site (50\%; Table 1$)$. There was one exposure, two implants showed clinically obvious folding (Fig. 2), and two showed remarkable induration. The total GRC rate for xenogenic implants in the abdominal wall was $30 \%$. Animals that had induration in their vaginal implants also showed induration of their abdominal implants.

In one vaginal explant, there was macroscopically no visible implant in between the fixation sutures any more. Vaginal and abdominal explants were of comparable thickness, though the contraction rate was almost three times higher in vaginal explants $(p=0.0008)$. There were significant differences between implantation sites for passive biomechanics: vaginal explants were $63 \%$ less stiff than their abdominal counterparts $(p=0.01)$, though the length of the comfort zone was comparable (Fig. 3a). Explants were also categorized by histologic signs of degradation. In the presence of histologic loss of implant integrity, degraded abdominal implants were more compliant than those without degradation (0.44 vs. 0.79 $\mathrm{N} / \mathrm{mm}$ ). Because of the low number, no statistics were done. For vaginal implants, the histology was taken from the anterior side. Of those animals with histologic signs of degradation, their corresponding posterior implants were compared to those without degradation. The compliance of posterior implants of animals with degradation in the anterior implant was lower than in those without ( 0.33 vs. $0.15 \mathrm{~N} / \mathrm{mm})$. Again, no statistics were done.

Histology of vaginal explants was scored on smaller anterior vaginal implants (Table 2). One must remember that this is a different location than the rectovaginal area described above. ACM abdominal explants had higher scores for FBGC $(p=0.0078)$ yet similar amounts of PMN and vascularization 
Table 1 Paired comparison of outcomes of vaginally and abdominally implanted ACMs

Biomechanical findings are displayed for all animals as well as results without the outlier, and contraction is displayed without those with unidentifiable or extruded material.

\begin{tabular}{llll}
\hline & ACM & \multirow{2}{*}{ Paired comparison } \\
\cline { 2 - 3 } & Abdomen & Posterior vagina & \\
\hline Graft-related complication & $3 / 10(30 \%)$ & $5 / 10(50 \%)$ & $\mathrm{ns}$ \\
$\quad$ Exposure & $0 / 10(0 \%)$ & $1 / 10(10 \%)$ & \\
Folding & $0 / 10(0 \%)$ & $2 / 10(20 \%)$ & \\
Induration & $3 / 10(30 \%)$ & $2 / 10(20 \%)$ & \\
Other gross anatomical findings & & & \\
Thickness (mm) & $8.22 \pm 3.90$ & $6.78 \pm 2.27$ & $\mathrm{~ns}$ \\
$\quad$ Material not identifiable & $0 / 10(0 \%)$ & $1 / 10(10 \%)$ & $\mathrm{ns}$ \\
Contraction of identifiable mesh & $-20.28 \% \pm 18.24$ & $-61.18 \% \pm 17.25$ & 0.0008 \\
Biomechanics & & & \\
All ewes & & & $\mathrm{ns}$ \\
Comfort zone stiffness $(\mathrm{N} / \mathrm{mm})$ & $0.68 \pm 0.2$ & $0.41 \pm 0.46$ & $\mathrm{~ns}$ \\
Comfort zone length $(\mathrm{mm})$ & $7.18 \pm 2.00$ & $8.52 \pm 3.22$ & \\
Exclusion of outlier $(n=9)$ & & & 0.0101 \\
$\quad$ Comfort zone stiffness $(\mathrm{N} / \mathrm{mm})(n=9)$ & $0.73 \pm 0.29$ & $0.27 \pm 0.19$ & $\mathrm{~ns}$ \\
Comfort zone length $(\mathrm{mm})(n=9)$ & $7.30 \pm 2.00$ & $8.90 \pm 3.17$ & \\
\hline
\end{tabular}

as vaginal ACM explants. Scores for collagen organization and composition were similar for both sites, but the amount of collagen was higher in the abdominal ACM explants $(p=0.0201)$. All the above scores were averages. At closer look, explants fall apart in two categories. In $70 \%$ of the anterior vaginal and $30 \%$ of the abdominal explants, we could not trace the typically dense structure of the ACM on histology (Fig. 2d, e). In these cases, there was no measurable inflammation but limited areas of organized and mature connective tissue. Isolated pockets of inflammation were visible only when a suture was accidentally caught in the section. In the other cases, the ACM could be identified. In that case, there was an abundance of inflammatory cells, both FBGC and PMN, as evidenced by their score, as well as connective tissue deposition. In five ACM sections, we observed a precipitated material compatible with calcification (Fig. 2c). Four were abdominal explants; three of them were macroscopically categorized as indurated. In one of these ewes, also the anterior and posterior vaginal explants were indurated. The anterior explant showed calcification on histology, which coincided with histologic signs of infection).

Vaginal contractility and comparison to PP vaginal implants

Figure 3 displays the vaginal contractility findings. Posterior ACM explants where no graft was recognizable on histology $(n=7)$ and the corresponding anterior vaginal implant had a $68 \%$ lower contractility than those with recognizable material ( $n=3$; Fig. $3 b)$. Again, in view of the very low numbers, no statistics were attempted.

We compared the vaginal contractility and other findings to observations made earlier when using a PP vaginal implant (Table 3). In the PP group, there was one case of GRC (folding), yet this apparently lower number was not significant. However, the $17 \%$ contraction rate of PP explants was significantly less than for ACM $(p=0.0009)$. Passive biomechanics of all vaginal ACM were comparable to observations in the PP explants. Compliance of degraded ACM $(0.33$ $\mathrm{N} / \mathrm{mm}$ ) was within the range of what was measured in PP $(0.29 \mathrm{~N} / \mathrm{mm})$ explants. Active biomechanical findings were also comparable. However, the values of three animals with recognizable ACM were in the range of the PP-implanted animals. Conversely, the forces generated by the seven animals without degradation in the anterior ACM were $60 \%$ lower than those of PP explants ( $p=0.048)$ (Fig. 3b).

The histology of vaginal explants with PP differed completely from those with recognizable ACM (Fig. 2f). PP induced a mild inflammation, with few cells, nearly all macrophages or FBGC, and less collagen deposition. These specimens showed no histologic signs of infection.

\section{Discussion}

In this study, we used the ovine model to document outcomes following insertion of a cross-linked ACM in the rectovaginal septum and, for histology, insertion of a smaller piece into the anterior vaginal wall. We used an investigational product that was treated with the ADAPT ${ }^{\circledR}$ procedure to prevent calcification, reduce lipid content and restore tissue flexibility. We used a double control, i.e. an internal control by implantation of the same material at a control site (the abdominal wall) and an external control by implantation of a durable PP implant at the same (vaginal) site. 


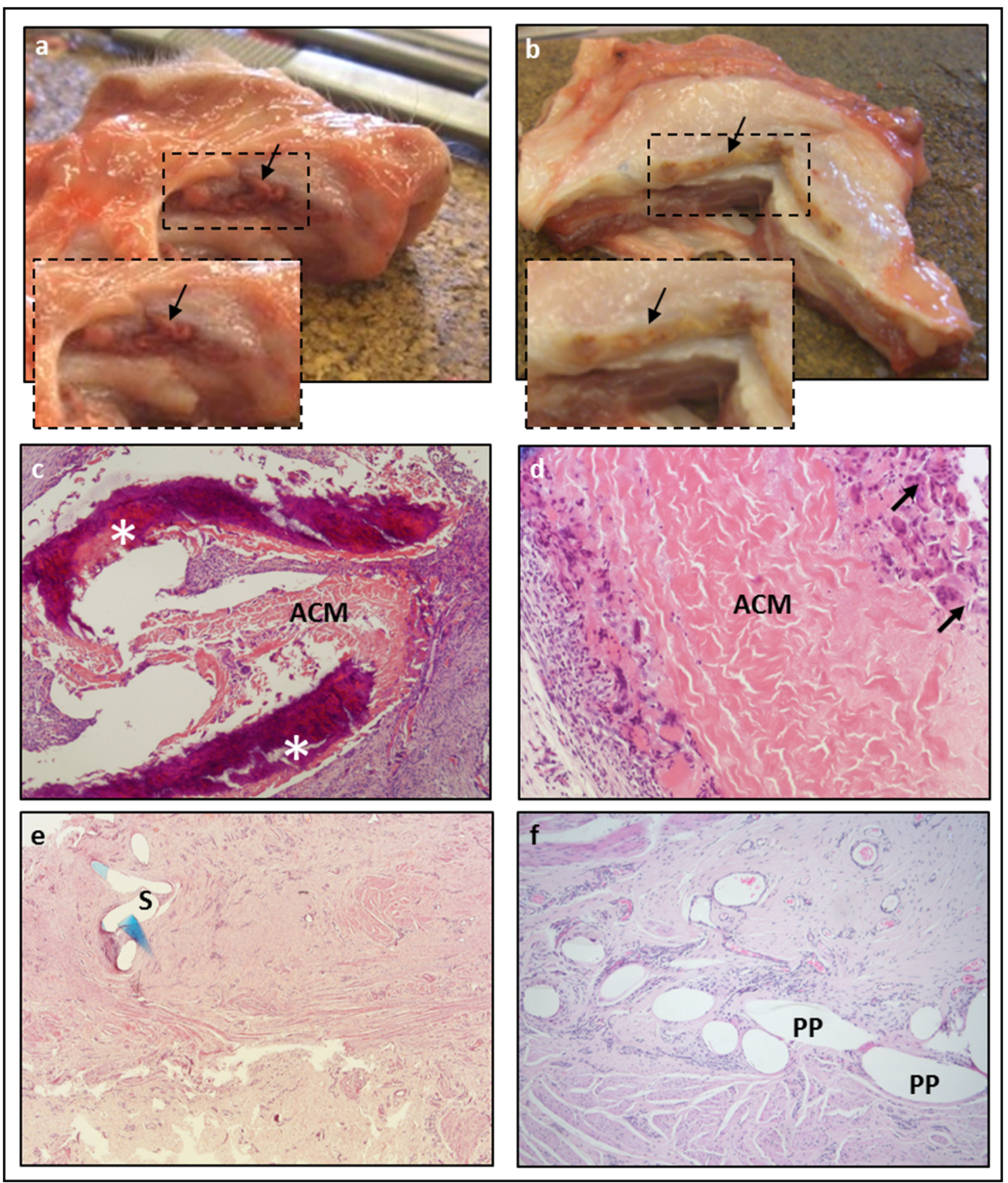

Fig. 2 Gross anatomy. Explants from the posterior vaginal wall (a) and the abdomen (b) with induration and folding visible in the below placed selections. The dark area (arrows) below the vaginal epithelium and above the abdominal muscles is the location of the implant, which looks complete. In these cases, the material was hard on palpation and surrounded with excessive amount of connective tissue. Histology

Conceptually, ACM is the ideal matrix for gradual integration into the host. In order to avoid adverse reactions, ACMs are made free of allergens, DNA and other pathogens. Crosslinking should make them resistant to endogenous collagenase activity. However, several experimental and clinical studies report unfavourable outcomes with the use of a variety of showed variable host response (c-e), gradual degradation of ACM with mineral precipitation or calcification (asterisk; c) and FBGC (arrow) on the graft-tissue interface (d), or complete ACM degradation where just sutures $(S)$ were identifiable (e). Polypropylene implant $(P P)$ showed uniform response (f). ( $\mathrm{H} \& \mathrm{E}, \times 40, \times 200, \times 25, \times 100$, respectively)

ACM. Clinically, both GRC and failure were reported [24-27]. Also, our experiment revealed a number of GRC. The first striking observation was the presence of calcification on histology. This happened at both anatomical locations and not in the control group. Calcification of GA cross-linked grafts has been tied to residual non-viable cells and cellular 


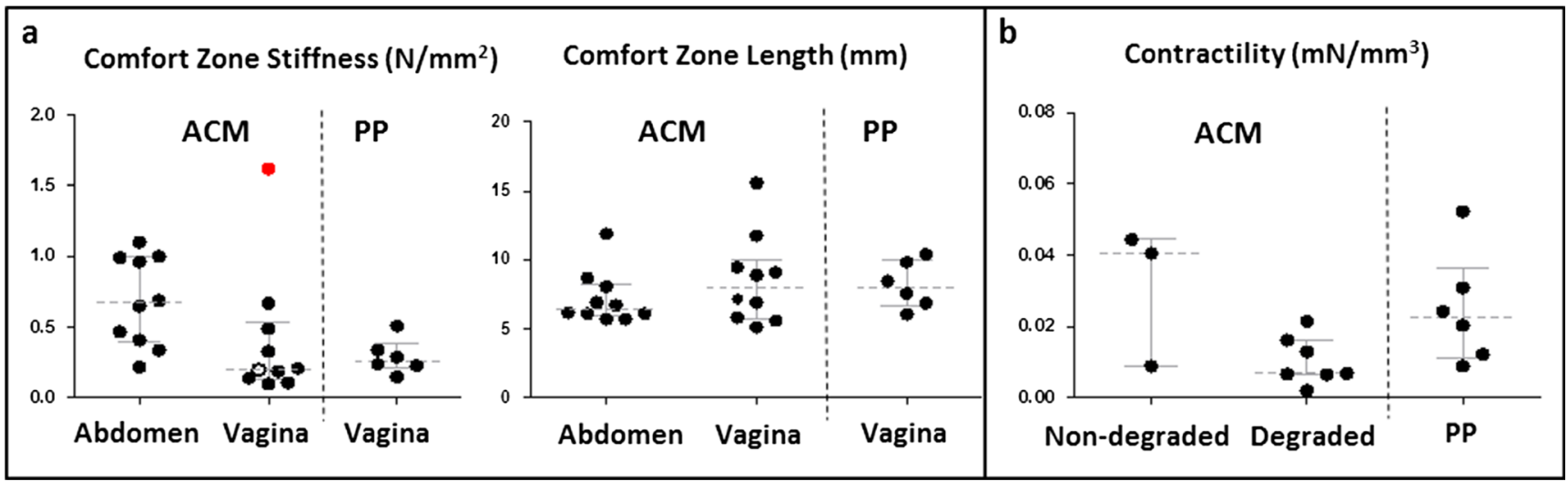

Fig. 3 Box plots and individual data of passive (a) and active (b) biomechanical tests. Individual data are plotted (black circle) with median and interquartile range marked with gray lines. The not identifiable implant is marked with an empty circle and outlier red circle

debris that were not removed during GAD pretreatment. They serve as nucleation sites for calcium phosphate minerals. GAD modifies phosphorous-rich cell membranes that are capable of mineralization using calcium present in the extracellular fluid $[28,29]$. This adverse effect was meant to be prevented by the ADAPT ${ }^{\circledR}$ procedure, which has been earlier shown to work in previous experimental studies in rats and sheep [30-32]. Clinical studies on the use of the same material for cardiac defects showed minimal or no mineral precipitation in the matrices [15]. This was reassuring because pathological calcification is a feared complication in cardiac surgery, certainly in young patients [33, 34]. Another identified risk factor for calcification of grafts is the occurrence of infection or folding $[35,36]$. These may add to other surgical factors, such as haemorrhage, or damage by surgical handling. To our knowledge, no previous studies have looked at the use of this material for surgical repair of the abdominal wall or a vaginal environment.

The most common local observation was palpable induration at the implant site. Histologically, this did not always coincide with calcification but may also with contraction, folding and wrinkling. There are no published data on contraction rates following insertion of cross-linked ACM in the vagina. Studies on abdominal insertion showed various results. Ozog et al. reported minimal or unchanged dimensions in 180 days with Pelvicol in rats, however, without visible deformation [37]. Conversely, Jenkins et al. reported a $50 \%$ area reduction and wrinkling for abdominal wall reconstruction after 3 months in mini pigs using Collamend (another cross-linked porcine dermis; Bard, Davol, RI) [38]. They explained the process by the presence of encapsulation, though we observed that process also in non-contracted ACM [37]. Contraction is, however, not the privilege of ACM, as significant contraction rates have been reported following PP implantation both in the vagina (45\%) and abdomen (10\%) 90 days after surgery [18].

We also observed various stages of graft degradation in $30 \%$ of abdominal and $70 \%$ of vaginal explants. The occurrence of degradation of cross-linked ACM has been reported by several groups in different models [39-42]. Claerhout et al. documented degradation in rabbit abdominal Pelvicol (Bard, Haasrode, Belgium) implants from 180 days onwards [40], and Pierce documented the same for vaginal Pelvisoft implants [41]. In a clinical study, we saw the same when Pelvicol was used for laparoscopic sacrocolpopexy, leading to recurrence [24]. Graft degradation coincided in 8 out of 9 cases with an abundance of foreign body (giant) cells on biopsy [43]. Description of the exact time course of degradation is currently impossible since clinical, as experimental studies usually involve only one time point. Interestingly, degradation does not occur in the same way at both locations. Pierce et al. showed more degradation of Pelvisoft (Bard, Covington, GA) 270 days following vaginal than abdominal implantation [44]. The same difference was present in our study. It is not possible to determine whether this is a faster or more vigorous process. However, Abramov et al. showed differences between the wound healing response in the vagina and in the abdomen, which may lead to another remodelling process [45].

Vaginal ACM explants were around 2.7 times less stiff than their abdominal counterparts. To our knowledge, there are no studies on biomechanics following simultaneous ACM implantation in both locations, so we do not have references to compare to. However, differences in biomechanics for these two environments were studied following insertion of PP (Gynemesh; Ethicon, Somerville, NJ) in a rabbit [44]. Vaginal explants were 1.5 less stiff than abdominal explants. Both observations follow a trend reported on biomechanics of cadaverous native tissue from the vagina and abdominal aponeurosis [46]. Gabriel et al. reported the vaginal wall being four times less stiff than abdominal aponeurosis. There are, to our knowledge, no published reference data on the biomechanics of native vaginal tissue and abdominal wall.

Graft degradation is another factor of relevance, as it interferes with the biomechanical properties. For that reason, we compared stiffness of those animals where no ACM was visible anymore versus those where the material was conserved. Effects seemed to be different according to the location of implantation. Yet, 
Table 2 Histological scores of host response and connective tissue formation following insertion of ACM and PP in the vagina and abdominal wall

\begin{tabular}{|c|c|c|c|c|c|}
\hline Histology & $\begin{array}{l}\text { Abdomen } \\
\text { ACM }\end{array}$ & Anterior vagina & Pair comparison & $\begin{array}{l}\text { Anterior vagina } \\
\text { PP }\end{array}$ & Unpaired comparison \\
\hline & $N=10$ & $N=10$ & & $N=6$ & \\
\hline FBGC & $0.8(0.88)$ & $0.1(0.53)$ & 0.0078 & $0.40(0.25)$ & \\
\hline PMN & $0.15(0.38)$ & $0.2(0.67)$ & & $0.05(0.23)$ & \\
\hline Vascularity & $1.0(0.4)$ & $1.15(0.3)$ & & $2.05(0.55)$ & 0.0023 \\
\hline Collagen organization & $1.5(1.4)$ & $1.4(0.35)$ & & $1.3(0.55)$ & \\
\hline Collagen composition & $1.8(0.4)$ & $2.1(0.4)$ & & $2.3(0.55)$ & \\
\hline Collagen amount & $2.7(0.2)$ & $2.5(0.2)$ & 0.0201 & $1.9(0.35)$ & 0.0061 \\
\hline Calcification & $4 / 10$ & $1 / 10$ & & $0 / 6$ & \\
\hline $\begin{array}{l}\text { Scores at the interface with identifiable } \\
\text { material on histology }\end{array}$ & $N=7$ & $N=3$ & & $N=6$ & \\
\hline FBGC & $1.1(0.7)$ & $0.8(0.35)$ & & $0.40(0.25)$ & \\
\hline PMN & $0.1(0.45)$ & $1.8(0.85)$ & & $0.05(0.23)$ & \\
\hline Vascularity & $0.7(0.45)$ & $1.5(0.45)$ & & $2.05(0.55)$ & \\
\hline Collagen organization & $1.4(1.0)$ & $0.8(0.5)$ & & $1.3(0.55)$ & \\
\hline Collagen composition & $1.8(0.3)$ & $2.4(0.3)$ & & $2.3(0.55)$ & \\
\hline Collagen amount & $2.8(0.3)$ & $2.8(0.1)$ & & $1.9(0.35)$ & \\
\hline
\end{tabular}

Scores are displayed for all explanted specimens and also for those with identifiable ACM. For later, statistics was not carried out due to a few specimens. Data for vaginal PP implants were previously published by Feola et al. 2014 [17]

$F B G C$ foreign body giant cell, $P M N$ polymorphonuclears

low numbers preclude actual statistical assessment, and more importantly, degradation was determined on the anterior implant, whereas biomechanics on the posterior implant. For those reasons, we prefer not to make any firm conclusions on this matter.

Smooth muscle contractility showed, at the first glance, no difference for the explants using different implant materials. Further analysis into two subgroups revealed a $68 \%$ decline of forces in group without visible ACM. When there was still detectable ACM, findings were comparable to PP-implanted tissues. In other words, the absence of material coincides with reduction in contractility. In line with other studies, we further demonstrated that ACM implants affect smooth muscle contractility [47]. This is somewhat counter-intuitive unless one assumes that earlier in time, the implant may have already compromised contractility, e.g. by a process of stress shielding, as described by others [47-49]. To elucidate that, one would, however, need a study with several time points and appropriate native tissue controls, and the mechanisms remain therefore unexplained.

Table 3 Outcomes and test results of vaginally implanted ACM and PP and their unpaired comparison (significance level $p<0.05$ )

\begin{tabular}{|c|c|c|c|}
\hline & $\begin{array}{l}\mathrm{ACM} \\
\text { Posterior vagina } \\
N=10\end{array}$ & $\begin{array}{l}\mathrm{PP} \\
\text { Posterior vagina } \\
N=6\end{array}$ & Unpaired comparison \\
\hline Graft-related complication & $5 / 10(50 \%)$ & $1 / 6(16.6 \%)$ & 0.0367 \\
\hline Exposure & $1 / 10(10 \%)$ & $0 / 6(0 \%)$ & \\
\hline Folding & $2 / 10(20 \%)$ & $1 / 6(16.6 \%)$ & \\
\hline Induration & $2 / 10(20 \%)$ & $0 / 6(0 \%)$ & \\
\hline Contraction of identifiable mesh & $-53.82 \% \pm 10.20(n=7)$ & $-23.06 \% \pm 16.63$ & 0.0009 \\
\hline \multicolumn{4}{|l|}{ Biomechanics } \\
\hline Comfort zone stiffness (N/mm) & $0.27 \pm 0.19(n=9)$ & $0.29 \pm 0.12$ & ns \\
\hline Comfort zone length (mm) & $8.89 \pm 3.17(n=9)$ & $8.18 \pm 1.67$ & ns \\
\hline Contractility $\left(\mathrm{mN} / \mathrm{mm}^{3}\right)$ & $0.017 \pm 0.015(n=10)$ & $0.025 \pm 0.016$ & ns \\
\hline Degraded $(n=7)$ & $0.010 \pm 0.007$ & $0.025 \pm 0.016$ & 0.0481 \\
\hline Non-degraded $(n=3)$ & $0.031 \pm 0.019$ & - & - \\
\hline
\end{tabular}


Our study has several limitations. Again, we only studied one time point, precluding insight into the time course of the host response; hence, it cannot learn on the processes driving the adverse events neither. Another is the use of a semiquantitative histological scoring system and limited staining methods. Though they are descriptive, it would be much more informative to also have biochemical and molecular read outs to document details on the nature of the immune response, neovascularization, collagen metabolism and nerve ingrowth [50]. This might be difficult to do, as those tools are not readily available in sheep and also add significantly to the cost. Further, we had to resort to use a second vaginal location for implantation so that we could obtain sufficient tissue for histology, without compromising the availability of tissue for biomechanical testing. Further, there are the limitations of the model; even though sheep can be used as a model for vaginal surgery as well as prolapse [18], it remains mostly an asymptomatic quadruped, with clinically different anatomical and pelvic floor loads, hence not an ideal disease model. It has however several advantages: apparently, sheep may clinically develop obvious pelvic floor relaxation and some degree of prolapse after delivery; further, this is a widely available, affordable alternative to the primate, which would be the closest model that we could think off.

In conclusion, we demonstrated that despite treatment with $\mathrm{ADAPT}^{\circledR}, \mathrm{ACM}$ insertion into the rectovaginal septum was associated with a number of local adverse effects. Apart from that, the passive biomechanical properties were not better than what was obtained when a PP mesh was used. Following degradation of the ACM, there was a decrease in smooth muscle contractility. The ideal implant material has apparently not yet been identified.

Acknowledgment We thank to Ivan Laermans, Rosita Kinart, Ann Lissens (Centre for Surgical Technologies, KU Leuven, Leuven, Belgium), Godelieve Verbist (Dept. of Development and Regeneration, KU Leuven, Leuven, Belgium) for their technical support during the experiment. We thank Leen Mortier for help with data and manuscript management.

Conflict of interest Masayuki Endo and Andrew Feola are recipients of a Marie Curie Industry-Academia Partnership Programme grant of the European Commission. They, as well as Iva Urbankova, Jaromir Vlacil, Siddarth Sengupta and Thomas Deprest, declare that they have no conflict of interest.

Bernd Klosterhalfen is a consultant for FEG Textiltechnik, Aachen, and a plaintiff's expert witness in the litigation case Cisson vs. Bard.

Jan Deprest is a fundamental clinical researcher of the Fonds Wetenschappelijk Onderzoek Vlaanderen (1801207). The experiment was partly supported by an unconditional grant from Blasingame, Burch, Garrard and Ashley (Atlanta, GA, USA) and the University of Western Australia. Agreements are handled via the Leuven Research and Development transfer office. Sponsors did not interfere with the planning, execution or reporting of this experiment neither are they the owner of the results.
Contribution to the authorship ME contributed to protocol development, experimental surgery, data collection and data analysis. IU contributed to histological analysis, data collection and data analysis. IU and JD were responsible for initial manuscript writing. JV and AF contributed to protocol development, experimental surgery, data collection and analysis. JV, SS, TD and BK contributed to histological analysis and data collection. All authors contributed to manuscript editing.

Ethical approval The experiment was approved by the Ethics Committee for Animal Experimentation of the Faculty of Medicine of the KU Leuven. All applicable international, national and institutional guidelines for the housing, care and use of animals were followed. Procedures performed in this study were in accordance with the ethical standards of the institution at which they were conducted.

Informed consent This article does not contain any studies with human participants.

Open Access This article is distributed under the terms of the Creative Commons Attribution License which permits any use, distribution, and reproduction in any medium, provided the original author(s) and the source are credited.

\section{References}

1. Barber MD, Maher C (2013) Epidemiology and outcome assessment of pelvic organ prolapse. Int Urogynecol J 24:1783-90. doi:10.1007/ s00192-013-2169-9

2. Milsom I, Altman D, Lapitan MC, et al. (2009) Epidemiology of urinary (UI) and faecal (FI) incontinence and pelvic organ prolapse (POP). 35-112.

3. Wu JM, Matthews CA, Conover MM et al (2014) Lifetime risk of stress urinary incontinence or pelvic organ prolapse surgery. Obstet Gynecol 123:1201-6. doi:10.1097/AOG.0000000000000286

4. Maher CM, Feiner B, Baessler K, Glazener CMA (2011) Surgical management of pelvic organ prolapse in women: the updated summary version Cochrane review. Int Urogynecol J 22:1445-57. doi:10. 1007/s00192-011-1542-9

5. Keys T, Campeau L, Badlani G (2012) Synthetic mesh in the surgical repair of pelvic organ prolapse: current status and future directions. Urology 80:237-43. doi:10.1016/j.urology.2012.04.008

6. Mangera A, Bullock A, Chapple CR, MacNeil S (2012) Are biomechanical properties predictive of the success of prostheses used in stress urinary incontinence and pelvic organ prolapse? A systematic review. Neurourol Urodyn 21:13-21. doi:10.1002/nau

7. Salomon LJ, Detchev R, Barranger E et al (2004) Treatment of anterior vaginal wall prolapse with porcine skin collagen implant by the transobturator route: preliminary results. Eur Urol 45:219-225. doi: 10.1016/j.eururo.2003.09.005

8. Meschia M, Pifarotti P, Bernasconi F et al (2007) Porcine skin collagen implants to prevent anterior vaginal wall prolapse recurrence: a multicenter, randomized study. J Urol 177:192-5. doi:10.1016/j.juro. 2006.08.100

9. Botros SM, Sand PK, Beaumont JL et al (2009) Arcus-anchored acellular dermal graft compared to anterior colporrhaphy for stage II cystoceles and beyond. Int Urogynecol J Pelvic Floor Dysfunct 20: 1265-71. doi:10.1007/s00192-009-0933-7

10. Mangera A, Bullock AJ, Roman S et al (2013) Comparison of candidate scaffolds for tissue engineering for stress urinary incontinence and pelvic organ prolapse repair. BJU Int 112:674-85. doi:10.1111/bju.12186

11. Trabuco EC, Klingele CJ, Weaver AL et al (2009) Medium-term comparison of continence rates after rectus fascia or midurethral sling 
placement. Am J Obstet Gynecol 200:300.e1-6. doi:10.1016/j.ajog. 2008.10.017

12. Jeon M-J, Jung H-J, Chung S-M et al (2008) Comparison of the treatment outcome of pubovaginal sling, tension-free vaginal tape, and transobturator tape for stress urinary incontinence with intrinsic sphincter deficiency. Am J Obstet Gynecol 199:76.e1-4. doi:10. 1016/j.ajog.2007.11.060

13. Dunn RM (2012) Cross-linking in biomaterials: a primer for clinicians. Plast Reconstr Surg 130:18S-26S. doi:10.1097/PRS. 0b013e31825efea6

14. Neethling WML, Glancy R, Hodge AJ (2004) ADAPT-treated porcine valve tissue (cusp and wall) versus Medtronic Freestyle and Prima Plus: crosslink stability and calcification behavior in the subcutaneous rat model. J Heart Valve Dis 13:689-96

15. Neethling WML, Strange G, Firth L, Smit FE (2013) Evaluation of a tissue-engineered bovine pericardial patch in paediatric patients with congenital cardiac anomalies: initial experience with the ADAPTtreated CardioCel(R) patch. Interact Cardiovasc Thorac Surg 17: 698-702. doi:10.1093/icvts/ivt268

16. Neethling WML, Hodge AJ, Clode P, Glancy R (2006) A multi-step approach in anti-calcification of glutaraldehyde-preserved bovine pericardium. J Cardiovasc Surg (Torino) 47:711-8

17. Feola A, Endo M, Urbankova I et al (2014) Host reaction to vaginally inserted collagen containing polypropylene implants in sheep. Am J Obstet Gynecol. doi:10.1016/j.ajog.2014.11.008

18. Manodoro S, Endo M, Uvin P et al (2013) Graft-related complications and biaxial tensiometry following experimental vaginal implantation of flat mesh of variable dimensions. BJOG 120:244-50. doi: 10.1111/1471-0528.12081

19. De Tayrac R, Alves A, Thérin M (2007) Collagen-coated vs noncoated low-weight polypropylene meshes in a sheep model for vaginal surgery. A pilot study. Int Urogynecol J Pelvic Floor Dysfunct 18:513-20. doi:10.1007/s00192-006-0176-9

20. Badylak S, Kokini K, Tullius B et al (2002) Morphologic study of small intestinal submucosa as a body wall repair device. J Surg Res 103:190-202. doi:10.1006/jsre.2001.6349

21. Zheng F, Lin Y, Verbeken E et al (2004) Host response after reconstruction of abdominal wall defects with porcine dermal collagen in a rat model. Am J Obstet Gynecol 191:1961-70. doi:10.1016/j.ajog. 2004.01.091

22. Morawietz L, Tiddens O, Mueller M et al (2009) Twenty-three neutrophil granulocytes in 10 high-power fields is the best histopathological threshold to differentiate between aseptic and septic endoprosthesis loosening. Histopathology 54:847-53. doi:10.1111/ j.1365-2559.2009.03313.x

23. Feola A, Moalli P, Alperin M et al (2011) Impact of pregnancy and vaginal delivery on the passive and active mechanics of the rat vagina. Ann Biomed Eng 39:549-58. doi:10.1007/s10439-010-0153-9

24. Claerhout F, De Ridder D, Roovers JP et al (2009) Medium-term anatomic and functional results of laparoscopic sacrocolpopexy beyond the learning curve. Eur Urol 55:1459-67. doi:10.1016/j.eururo. 2008.12.008

25. Deprest J, De Ridder D, Roovers J-P et al (2009) Medium term outcome of laparoscopic sacrocolpopexy with xenografts compared to synthetic grafts. J Urol 182:2362-8. doi:10.1016/j.juro.2009.07.043

26. Quiroz LH, Gutman RE, Shippey S et al (2008) Abdominal sacrocolpopexy: anatomic outcomes and complications with Pelvicol, autologous and synthetic graft materials. Am J Obstet Gynecol 198:557.e1-5. doi:10.1016/j.ajog.2008.01.050

27. Hviid U, Hviid TVF, Rudnicki M (2010) Porcine skin collagen implants for anterior vaginal wall prolapse: a randomised prospective controlled study. Int Urogynecol J 21:529-34. doi:10.1007/s00192009-1018-3

28. Jorge-Herrero E, Garcia Paez JM, Del Castillo-Olivares Ramos JL (2005) Tissue heart valve mineralization: review of calcification mechanisms and strategies for prevention. J Appl Biomater Biomech 3:67-82

29. Schoen FJ, Levy RJ (2005) Calcification of tissue heart valve substitutes: progress toward understanding and prevention. Ann Thorac Surg 79:1072-80. doi:10.1016/j.athoracsur.2004.06.033

30. Van den Heever JJ, Neethling WML, Smit FE et al (2013) The effect of different treatment modalities on the calcification potential and cross-linking stability of bovine pericardium. Cell Tissue Bank 14: 53-63. doi:10.1007/s10561-012-9299-z

31. Neethling WML, Glancy R, Hodge AJ (2010) Mitigation of calcification and cytotoxicity of a glutaraldehyde-preserved bovine pericardial matrix: improved biocompatibility after extended implantation in the subcutaneous rat model. J Heart Valve Dis 19:778-85

32. Neethling WML, Yadav S, Hodge AJ, Glancy R (2008) Enhanced biostability and biocompatibility of decellularized bovine pericardium, crosslinked with an ultra-low concentration monomeric aldehyde and treated with ADAPT. J Heart Valve Dis 17:456-63

33. Schoen FJ, Hobson CE (1985) Anatomic analysis of removed prosthetic heart valves: causes of failure of 33 mechanical valves and 58 bioprostheses, 1980 to 1983. Hum Pathol 16:549-59

34. Butany J, Leong SW, Cunningham KS et al (2007) A 10-year comparison of explanted Hancock-II and Carpentier-Edwards supraannular bioprostheses. Cardiovasc Pathol 16:4-13. doi:10. 1016/j.carpath.2006.06.003

35. Schoen FJ (1987) Biomaterial-associated infection, neoplasia, and calcification: clinicopathologic features and pathophysiologic concepts. ASAIO Trans 33:8-18

36. Cunanan C, Cabiling C, Dinh T (2001) Tissue characterization and calcification potential of commercial bioprosthetic heart valves. Ann Thorac Surg 417-421

37. Ozog Y, Konstantinovic M, Zheng F et al (2009) Porous acellular porcine dermal collagen implants to repair fascial defects in a rat model: biomechanical evaluation up to 180 days. Gynecol Obstet Invest 68:205-12. doi:10.1159/000235852

38. Jenkins ED, Melman L, Deeken CR et al (2011) Biomechanical and histologic evaluation of fenestrated and nonfenestrated biologic mesh in a porcine model of ventral hernia repair. J Am Coll Surg 212:32739. doi:10.1016/j.jamcollsurg.2010.12.006

39. Deeken CR, Melman L, Jenkins ED et al (2011) Histologic and biomechanical evaluation of crosslinked and non-crosslinked biologic meshes in a porcine model of ventral incisional hernia repair. J Am Coll Surg 212:880-8. doi:10.1016/j.jamcollsurg.2011.01.006

40. Claerhout F, Verbist G, Verbeken E et al (2008) Fate of collagenbased implants used in pelvic floor surgery: a 2-year follow-up study in a rabbit model. Am J Obstet Gynecol 198:94.e1-94.e6

41. Pierce LM, Rao A, Baumann SS et al (2009) Long-term histologic response to synthetic and biologic graft materials implanted in the vagina and abdomen of a rabbit model. Am J Obstet Gynecol 200: 546.e1-8. doi:10.1016/j.ajog.2008.12.040

42. Melman L, Jenkins ED, Hamilton NA et al (2011) Early biocompatibility of crosslinked and non-crosslinked biologic meshes in a porcine model of ventral hernia repair. Hernia 15:157-64. doi:10.1007/ s10029-010-0770-0

43. Deprest J, Klosterhalfen B, Schreurs A et al (2010) Clinicopathological study of patients requiring reintervention after sacrocolpopexy with xenogenic acellular collagen grafts. J Urol 183: 2249-55. doi:10.1016/j.juro.2010.02.008

44. Pierce LM, Grunlan MA, Hou Y et al (2009) Biomechanical properties of synthetic and biologic graft materials following long-term implantation in the rabbit abdomen and vagina. Am J Obstet Gynecol 200:549.e1-8. doi:10.1016/j.ajog.2008.12.041

45. Abramov Y, Golden B, Sullivan M et al (2007) Histologic characterization of vaginal vs. abdominal surgical wound healing in a rabbit model. Wound Repair Regen 15:80-6. doi:10.1111/j.1524-475X. 2006.00188.x 
46. Gabriel B, Rubod C, Brieu M (2011) Vagina, abdominal skin, and aponeurosis: do they have similar biomechanical properties? Int Urogynecol J 22:23-27. doi:10.1007/s00192-010-1237-7

47. Feola A, Abramowitch S, Jallah Z et al (2013) Deterioration in biomechanical properties of the vagina following implantation of a highstiffness prolapse mesh. BJOG 120:224-32. doi:10.1111/1471-0528. 12077

48. Majima T, Yasuda K, Tsuchida T et al (2003) Stress shielding of patellar tendon: effect on small-diameter collagen fibrils in a rabbit model. J Orthop Sci 8:836-41. doi:10.1007/s00776-0030707-X

49. Lo IKY, Marchuk L, Majima Tet al (2003) Medial collateral ligament and partial anterior cruciate ligament transection: mRNA changes in uninjured ligaments of the sheep knee. J Orthop Sci 8:707-13. doi: 10.1007/s00776-003-0695-x

50. Liang R, Zong W, Palcsey $\mathrm{S}$ et al (2014) Impact of prolapse meshes on the metabolism of vaginal extracellular matrix in rhesus macaque. Am J Obstet Gynecol. doi:10.1016/j.ajog.2014.08.008 